\title{
Urinary diversions for radical cystectomy: a review of complications and their management
}

\author{
Catarina Laranjo Tinoco ${ }^{1}$, Estevão Lima ${ }^{2,3}$ \\ 'Urology Department, Hospital de Braga, Braga 4710-243, Portugal. \\ ${ }^{2}$ Life and Health Sciences Research Institute, ICVS/3B's - Associate Lab, School of Medicine - University of Minho, Braga 4710- \\ 057, Portugal. \\ ${ }^{3}$ CUF Urology, CUF Hospital, Lisbon 1350-352, Portugal.
}

Correspondence to: Dr. Catarina Laranjo Tinoco, Urology Department, Hospital de Braga, Sete Fontes - São Victor, Braga 4710243, Portugal. E-mail: cat.tinoco@gmail.com

\begin{abstract}
How to cite this article: Tinoco CL, Lima E. Urinary diversions for radical cystectomy: a review of complications and their management. Mini-invasive Surg 2021;5:28. https://dx.doi.org/10.20517/2574-1225.2021.35
\end{abstract}

Received: 14 Mar 2021 First Decision: 22 Mar 2021 Revised: 28 Mar 2021 Accepted: 12 Apr 2021 Available online: 6 Jun 2021

Academic Editor: Giulio Belli Copy Editor: Xi-Jun Chen Production Editor: Xi-Jun Chen

\begin{abstract}
Radical cystectomy involves a urinary diversion, the most used being the ileal conduit and the orthotopic neobladder. This review focuses on the complications associated with these procedures, dividing them into general and diversion related complications, as well as their management. We conducted a search on PubMed and Scopus to identify eligible articles on complications of urinary diversions. Randomized controlled trials and systematic reviews with meta-analysis were preferred when available. Early complications occur in the first 90 days after surgery. The most common is post-operative ileus, followed by urinary tract infections and urinary leakage. Most complications occur in the late post-operative setting, being related to the type of urinary diversion. Some of these complications are renal failure, metabolic abnormalities, infections, urolithiasis, and ureteroenteric strictures, each with particular management options. Specific ileal conduit complications are conduit deformities and parastomal hernias. Neobladder patients can have continence problems, like incontinence or urinary retention, but also fistulas and dehiscence. Standardization of complications' definitions and time-dependent reporting are crucial to better understand and manage these complications. Complication rates are similar between open and robot-assisted procedures and between intracorporeal and extracorporeal diversion. Radical cystectomy with urinary diversion is the most difficult surgical procedure in urology with high early and late complication rates. There is an urgent need of standardizing complication reporting to better compare different procedures.
\end{abstract}


Keywords: Urinary diversion, ileal conduit, orthotopic neobladder, cystectomy, urinary bladder neoplasms, complication

\section{INTRODUCTION}

Radical cystectomy for the treatment of bladder cancer implies a urinary diversion for replacement of the lower urinary tract, which nowadays can be created in an extracorporeal or minimally invasive totally intracorporeal way. The ideal urinary reservoir would be a low-pressure system, storing approximately 500 $\mathrm{mL}$ of urine, with complete continence, complete voluntary control of voiding, and minimal absorption of urinary waste products ${ }^{[1]}$. The variety of urinary diversion types underlines the absence of an ideal one. They can be divided into noncontinent and continent diversions. Noncontinent cutaneous diversions include cutaneous ureterostomies and bowel conduits; continent diversions can be cutaneous, with a catheterizable pouch, or orthotopic, as the famous neobladder. The most used urinary diversions are the ileal conduit and the orthotopic neobladder, which will be the focus of this review. Both have specific complications which will be discussed, as well as their management. They can be created by an extracorporeal open approach or in a minimally invasive totally intracorporeal way, with similar complications. The complication rates described in this review are summarized in Table 1. Comparing diversions is beyond the scope of this review.

\section{METHODS}

A search using the keywords "radical cystectomy", "urinary diversion", "neobladder", "ileal conduit", and "complications" was conducted on PubMed and Scopus to identify eligible articles. We focused primarily on randomized clinical trials and systematic reviews/meta-analysis when available, but we mostly included retrospective studies, case series, and case reports. We also used the "snowball method", involving tracking references of the previously chosen articles to identify additional relevant studies. Only articles in English, Portuguese, and Spanish were reviewed.

\section{GENERAL COMPLICATIONS}

\section{Early post-operative complications (90 days)}

Surgical morbidity is always dependent on correct reporting of the complications, and radical cystectomy with urinary diversion rates is an area where this is particularly evident. Studies show a wide range of early post-operative complication during the first 90 days (20\%-80.5\%), of both open or robot-assisted radical cystectomy (RARC) $)^{[-5]}$. The lack of standardized complication definitions may be one explanation for this discrepancy.

Gastrointestinal complications like ileus or small bowel obstruction and infectious complications are the most frequent ${ }^{[2-4,6]}$. The "robot-assisted radical cystectomy $v s$. open radical cystectomy in patients with bladder cancer" (RAZOR) clinical trial showed no differences between early complication rates for open (67\%) vs. robot-assisted procedures (69\%), even when only major complications were considered. All the urinary diversions in the RAZOR trial were performed extracorporeally, which can influence complication results ${ }^{[6]}$. However, performing the urinary diversion in an extracorporeal or intracorporeal way also carries similar complication rates, with a trend towards less gastrointestinal complications in the intracorporeal urinary diversion ${ }^{[7,8]}$. Early complications are less related to the type of urinary diversion than late complications ${ }^{[3]}$.

Ileus

Post-operative ileus can have a multitude of definitions, but the most used is "the inability to tolerate solid 
Table 1. Overall complication rates reported by the cited articles

\begin{tabular}{llll}
\hline & General complications & \multicolumn{1}{c}{ Specific complications } \\
\hline Early (90 days) & & Ileal conduit & $11 \%-17.1 \%$ \\
\hline lleus & $12 \%-23 \%$ & Parastomal hernia & \\
Urinary tract infection & $5.7 \%-44 \%$ & Conduit deformities & $2.4 \%$ \\
Urinary leakage & & Strictures & $<1 \%$ \\
Uretero-ileal & $2 \%-5.5 \%$ & Neobladder & $<2 \%$ \\
Urethral & $<25.3 \%$ & Rupture & $2.7 \%-8.8 \%$ \\
Late & & Fistula & \\
Renal failure & $19 \%$ & Neobladder-enteric & $4 \%-8 \%$ \\
Metabolic abnormalities & $<50 \%$ & Neobladder-vaginal & $24 \%-62.5 \%$ \\
Acidosis & $10.2 \%-33 \%$ & Hypercontinence & $3 \%-43 \%$ \\
B12 deficiency & $3 \%$ & Male & $<54.7 \%$ \\
Urolithiasis & $3.5 \%-15.3 \%$ & Female & Incontinence \\
Ureteroenteric stricture & $1.3 \%-10 \%$ & Daytime & Nightime \\
& & &
\end{tabular}

food by post-operative day 5 , the need to place a nasogastric tube (NGT), or the need to stop oral intake due to abdominal distension, nausea, or emesis". In a study by Shabsigh et al. ${ }^{[3]}, 23 \%$ of patients suffered from ileus by this definition. Hautmann et al. ${ }^{[4]}$ also used this definition to report an ileus rate of $12 \%$ in a large neobladder series of 1013 patients. In the RAZOR trial, the rate of ileus in open and robotic cystectomy was similar, $20 \%$ and $22 \%$, respectively ${ }^{[6]}$. A comparison between extracorporeal and intracorporeal approaches showed less time to return of bowel activity in intracorporeal robot-assisted diversions, which the authors attribute to less pain and analgesic use and faster return to normal activity due to smaller and less painful incisions and to less ambient air exposure of the peritoneum and abdominal viscera ${ }^{[8]}$.

Enhanced recovery after surgery (ERAS) protocols for cystectomy play an important role in reducing postoperative ileus, with a multimodal approach to prevent this complication which frequently prolongs hospitalization. Chewing gum and post-operative nasogastric tube avoidance, for example, seem to be effective in reducing ileus ${ }^{[9,10]}$.

\section{Urinary tract infection}

Urinary tract infections (UTIs) are a common complication causing readmission in many cystectomized patients. Early post-operative UTI rates range from $5.7 \%$ to $44 \%$, but a lack of standardization is evident, and the rates greatly depend on the UTI definition. Diagnosing a UTI in a patient with a urinary diversion requires a high level of suspicion because of its vague presentation, ranging from abdominal discomfort and changes in urine's smell to septic shock. Most often UTIs occur before stent removal, and their higher frequency in the early $v s$. late post-operative period also suggests an important role of these foreign bodies in UTI pathogenesis. Antibiotic treatment should be directed to cultured microorganisms as soon as possible $e^{[11-13]}$.

Clifford et al. ${ }^{[11]}$ reported a global rate of $11 \%$ patients develop UTIs in the first 90 days post-operatively, predominantly by Gram-negative rods; $17 \%$ of those patients had recurrent infections and $20 \%$ had urosepsis. They studied UTI rate by type of urinary diversion and found no significative difference between diversion types (orthotopic neobladder, continent cutaneous diversion, and ileal conduit) ${ }^{[1]}$. On the other hand, other studies found UTIs to be more frequent in orthotopic neobladder than in heterotopic 
diversions $^{[12,13]}$.

Urinary leakage

Ileal conduit ureteroileal anastomosis leak occurs in $2 \%-5.5 \%$ of the patients in the short term. Urethral anastomotic leaks in orthotopic neobladders are more frequent, reaching a rate of $25 \%$ in the first 90 days. In a case series by Nazmy et al. ${ }^{[2]}$, of the RARCs with neobladder, $25.3 \%$ had urethral leaks but most were minor and only $7.7 \%$ had a leak requiring catheterization, in line with previous reported rates. Treatment is most frequently conservative ${ }^{[2]}$.

\section{Late post-operative complications}

As studies in urinary diversion complications usually focus on early complications due to the high early mortality of the underlying cancer, with cancer-specific survival rates of $66 \%$ at 5 years $^{[14]}$, high-quality information on long-term complications is sparse. Long-term complications are most frequently related to the urinary diversion itself than the extirpative radical cystectomy. A large series of conduit patients (1057 patients) reported a high long-term complication rate of almost $80 \%$ at 20 years but a low reintervention rate of $6 \%{ }^{[15]}$. In their series of about 1000 patients with ileal neobladder followed over 25 years, Hautmann et al. ${ }^{[16]}$ report a long-term complication rate of $40.8 \%$, mostly diversion-related, with 3 neobladder-related deaths. They underline the importance of standardized reporting of long-term complications in a timedependent matter, explaining that only this way investigators can stop underrepresenting late complications since the number of patients decreases with time ${ }^{[16]}$. However, studies with this methodology are still lacking, so most of the following complication rates are still calculated on a non-time-dependent matter.

\section{Renal failure}

New onset of renal failure occurs in $19 \%$ at a median of 2.3 years, with $2.5 \%$ progressing to renal replacement therapy at a median of 8.4 years $^{[15]}$. This is intimately related to ureteroenteric stenosis causing hydronephrosis, which will be reviewed below, and also to chronic infection and reflux of infected urine. It remains to be clarified if this loss of renal function is greater than the expected age-related deterioration. Careful follow-up is needed.

\section{Metabolic abnormalities}

Metabolic complications are linked to the intestinal shortening, the bowel segment resected and the absorptive properties of the conduit or neobladder intestinal mucosa. Acid-base disorders, vitamin deficiencies and electrolyte disturbances are consistently reported in the literature. The most frequent $\mathrm{pH}$ disturbance is metabolic hyperchloremic acidosis due to chloride absorption and bicarbonate excretion, especially if a colonic segment is used. Vitamin $B_{12}$ deficiency is also expected, as this vitamin is absorbed in the terminal ileum, a segment frequently resected to use both in conduits and neobladders. This hypovitaminosis is mainly asymptomatic but can evolve to megaloblastic anemia, neuropathy, glossitis, and other diseases after the body's stores are depleted, which usually last 3-5 years ${ }^{[17]}$.

A large series of intestinal conduit diversion patients described $10.2 \%$ of metabolic acidosis requiring alkalinizing treatment and $3 \%$ of vitamin $\mathrm{B}_{12}$ deficiency occurring after a median of 9 years after surgery ${ }^{[15]}$. In continent diversions, long-term metabolic abnormalities can occur in as high as $50 \%$ of the patients ${ }^{[1]}$. In a neobladder sample, metabolic acidosis was diagnosed in the early post-operative period but $33 \%$ of the patients needed alkalinizing therapy for longer than 1 year and $1 \%$ of the patients were rehospitalized due to the acid-base imbalance ${ }^{[16]}$. 
Acidosis may be managed with alkalinizing treatment with sodium bicarbonate and vitamin deficiency with oral or parenteral supplementation ${ }^{[15-17]}$.

\section{UTI}

Risk factors for UTIs are incomplete emptying of urinary pouch (as residual urine is an infectious focus), intermittent catheterization or stenosis of the stoma or ureterointestinal anastomosis. Bacteriuria is common in these patients, but UTIs and urosepsis are not, so there is no need for prolonged suppressive antibiotic therapy. Although less frequent than in the early post-operative period, UTI in this setting should also be treated with a short course of antibiotics ${ }^{[16]}$.

\section{Urolithiasis}

The bowel epithelium, incomplete emptying of reservoirs with urinary stasis, foreign materials like staples, and chronic bacterial colonization or UTIs all contribute to stone formation, not uncommon in these patient ${ }^{[17]}$. These stones are mostly infectious and mixed, with metabolic stones being less frequent, particularly if only reservoir stones are considered ${ }^{[18]}$.

Regarding the role of staples in stone formation, Muto et al. ${ }^{[19]}$ reviewed their series of stapled neobladders and report a global stone rate of $4.6 \%$, with a risk of stone formation of $4.5 \%, 6.5 \%, 8.5 \%$, and $10 \%$ at 5,10 , 15 , and 20 years, respectively. They highlight the role of synchronous risk factors such as outlet obstruction and UTI in these patients and note that when they treated the stones endoscopically, the stapled lines were usually completely covered by mucosa ${ }^{[19]}$.

A study on conduit recipients reported a stone rate of $15.3 \%$ at a median of 2.5 years, more frequently in the upper urinary tract than in the conduit; less than $20 \%$ required treatment ${ }^{[15]}$. Marien et al ${ }^{[18]}$ studied 99 patients with urolithiasis after urinary diversion (not exclusively oncologic patients) and report an equal rate of upper and lower urinary tract stones, including 15 patients with both. The rates of urolithiasis in a recent meta-analysis were $3.5 \%$ for ileal conduits and $6.4 \%$ for neobladders, with a statistically significant difference ${ }^{[20]}$. Treatment options include all classical options for urolithiasis treatment, but endourological procedures and external lithotripsy are preferred ${ }^{[15]}$.

\section{Ureteroenteric stricture}

All but the cutaneous ureterostomy diversion involve uretero-enteric anastomoses. There are multiple techniques for anastomosing the ureters to the bowel, either refluxing or nonrefluxing.

The stricture of this anastomosis is a well-recognized complication with its serious consequences being the deterioration of the glomerular filtration rate with loss of kidney function. The rates of stenosis described in the literature range from $1.3 \%$ to $10 \%$, occurring predominantly on the first 2 years after surgery ${ }^{[2,21]}$.

Ureteroenteric stricture can have malignant causes, but most are benign. The pathophysiology of the benign stricture formation is not fully understood but it is likely secondary to ischemia or urine leakage leading to periureteral fibrosis. Preserving the ureteral blood supply, with careful handling and minimization of electrocautery around the ureters, and the creation of tension-free anastomoses may reduce the stenosis risk. Excision of redundant ureteric length, wider anastomosis, using stents for protection and testing with saline for leaks are other recommendations that can reduce the rate of this complication ${ }^{[21]}$. The use of intraoperative indocyanine green (ICG) fluorescence to evaluate ureteric vascularity and choose the site of ureteric division may reduce the risk of stricture; this is specially used in RARC, using the camera's capabilities $^{[22]}$. 
After development of a stricture, the patients can complain of flank pain or present with recurrent UTIs or urinary stones. If they develop an acute obstruction, decompression with a percutaneous nephrostomy is required. Nevertheless, a significant proportion will be asymptomatic, with diagnosis of ureteroenteric stenosis after imaging exams incidentally revealing hydronephrosis or blood tests hinting a deterioration of renal function.

The open repair of the stenosis with excision of the affected segment and reimplantation of the ureter is the gold-standard treatment, with the greatest rates of long-term success (up to 80\%), but it involves high technical expertise as these patients frequently have adhesions from the previous surgery. Therefore, minimally invasive options are being increasingly used.

Endourological access to the stenosis can be retrograde or anterograde through a percutaneous nephrostomy. Treatment may involve stenting, balloon dilation, or endoureterotomy (using cold knife, laser, or other devices); balloon dilation is the less effective method. These techniques have reported success rates of $4 \%-50 \%$.

Although achieving less long-term patency when compared to open revision, endourological techniques have less morbidity, shorter operative times and post-operative recovery and reduced costs, what makes them an attractive option. In general, endoscopic techniques are recommended as a first-line treatment for short strictures $(\leq 1 \mathrm{~cm})$ and for patients who cannot stand open repair ${ }^{[2,23]}$.

Minimally invasive alternatives to open revision, such as laparoscopic and robot-assisted repair, seem to achieve similar results with less morbidity ${ }^{[24,25]}$. All these techniques are useful in managing this frequent complication, when carefully selected.

\section{DIVERSION SPECIFIC COMPLICATIONS}

Each diversion type has its own specific complication, related not only to the construction of the diversion but also to the chosen intestinal segment. The surgical approach (open $v s$. robot-assisted and extracorporeal $v s$. intracorporeal) is not related to specific complications ${ }^{[6,7]}$.

\section{lleal conduit}

Parastomal hernia

Parastomal hernia (PSH) definition can be clinical or radiographic, with substantial heterogeneity across studies. The largest systematic review to date, which included only retrospective observational studies, used a clinical definition of a palpable bulge at the base of stoma and a radiographic definition of a crosssectional image evidencing protrusion of intraabdominal contents through the abdominal wall defect created to fixate the conduit. Of the total of 3170 patients submitted to radical cystectomy with ileal conduit, $529(17.1 \%)$ developed a PSH based on those criteria. The authors point that a substantial number of PSH remains asymptomatic and are only detected in the oncologic follow-up imaging studies ${ }^{[26]}$.

Treatment of PSH may be needed to alleviate symptoms such as pain or poor fit of ostomy bags or because of more serious complications like bowel obstruction or strangulation. Conservative treatment with the use of a hernia belt is a possibility, but no studies reported outcomes related to this modality. Surgical correction for PSH is frequently avoided due to the difficulty of the technique, high morbidity, and frequent hernia recurrence. Common procedures include local repair, the use of a synthetic or biological mesh, or relocating the stoma, mainly based on general surgery literature. A technique of local repair of PSH after ileal conduit was described by Rodriguez Faba et al. ${ }^{[27]}$ : a ipsilateral relocation of stoma without the need of midline 
incision, reducing the surgical risks. The global recurrence rates following surgical treatment of PSH in the largest systematic review were $27 \%-50 \%{ }^{[26]}$.

Another approach to PSH is prevention. The first randomized controlled study on the prevention of PSH after ileal conduit urinary diversion following radical cystectomy has been published with promising results. The authors concluded that the addition of a prophylactic sublay mesh decreases the risk of PSH, with incidences at 24 months of $11 \%$ in the intervention group compared to $23 \%$ in the control group. There was a small increase in median intraoperative time of $\sim 50 \mathrm{~min}$ and no greater risk of mesh-related complications. As an increased BMI was also associated with a higher risk of PSH in this study, the authors recommend the use of this prophylactic measure especially in obese patients. Studies with longer follow-up periods and health-economic analysis can clarify the role of the prophylactic mesh in a wider population ${ }^{[28]}$.

\section{Conduit deformities}

Conduit stricture and conduit elongation are possible complications of this diversion, poorly defined and reported in the literature. Shimko et al. ${ }^{[15]}$ reported conduit strictures in $2.4 \%$ of patients at a median of 9.4 years. These complications are reportedly less frequent than stomal complications.

\section{Orthotopic neobladder}

Rupture

Neobladder rupture is a rare but feared complication, possibly life-threatening. In large series of neobladder patients by Hautmann et al. ${ }^{[16]}, 3$ in 923 patients had neobladder rupture. Possible causes are perforation during catheterization, external trauma like car accidents, or outlet obstruction by a mucous plug. It can also happen spontaneously, for example in acutely or chronically overdistended reservoirs with wall ischemia or in patients previously submitted to pelvic irradiation. Timed micturition and post void residual volume surveillance (either with echography or catheterization) are advised in order to prevent this complication. In the event of rupture, these patients often present with an acute abdomen and need a relaparotomy to drain the urinoma and to repair the perforation or construct a substitute diversion. Alternatively, there are some descriptions of a conservative approach with neobladder catheterization, bilateral nephrostomies, and abdominal drain, in strictly selected patients with a mild presentation and hemodynamic stability ${ }^{[29]}$.

\section{Fistula}

Urinary diversion-enteric fistula is a rare complication, occurring in less than $2 \%$ of the patients submitted to radical cystectomy. Few studies are published on this complication and most involve neobladder patients. The most common and characteristic presentation is fecaluria, but pneumaturia and recurrent UTIs are other complaints. Diagnostic investigation with a CT usually shows air in the intestinal reservoir; if oral contrast is administered, it can appear in the urinary system. Msezane et al. ${ }^{[30]}$ report the location of the fistula to be more frequently from the small bowel anastomosis to the anterior neobladder wall. Small fistulas can be treated conservatively, with total parenteral nutrition, bladder drainage and treatment of sepsis, if present. For bigger fistulas or in cases of conservative treatment failure, open repair is the gold standard.

In women submitted to vaginal-sparing radical cystectomy with orthotopic neobladder creation, a neobladder-vaginal fistula can form in $2.7 \%-8.8 \%$, result of a much thinner neobladder wall ${ }^{[31]}$. Injury to the vaginal wall during dissection of the posterior bladder wall and urethra is an important risk factor, in which case conversion to other urinary diversion may be advisable ${ }^{[32]}$. Omental flap interposition between the neobladder and the vaginal stump during cystectomy or avoidance of overlapping suture lines are 
preventive measures, although it may not always prevent fistulization ${ }^{[31]}$.

These patients present with urinary incontinence and the fistula can be confirmed by cystoscopy or voiding cystography. Conservative management is invariably unsuccessful. The initial treatment option is a multilayered fistula closure via a transvaginal approach ${ }^{[31]}$; interposition with a Martius flap is an alternative, particularly important in recurrent fistulas ${ }^{[3,3,3]}$. In case of failure of the transvaginal approach, a transabdominal approach or conversion to a cutaneous diversion may be needed ${ }^{[31]}$.

\section{Hypercontinence}

Failure to empty the neobladder and urinary retention is much more frequent in women than in men. Neobladder patients with emptying failure can present with urinary retention but also with recurrent UTIs, hydronephrosis, or overflow incontinence.

The risk of retention increases with time, and emptying failure rates range from $4 \%$ to $8 \%$ in men and $24 \%$ to $62.5 \%$ in women ${ }^{[35,36]}$.

The cause of urinary retention in female neobladder recipients is still controversial. The literature ascribes the retention in women more to mechanical factors than functional or neurogenic ones; an explanation given for this chronic retention is a urethral "kinking" by prolapse of the vaginal stump with herniation of the posterior pouch through the anterior vaginal wall, due to lack of proper back support. Ali-El-Dein et al ${ }^{[37]}$ focused on this matter and defined chronic retention by a post-void residue of $20 \%$ of mean maximal pouch capacity (approximately $100 \mathrm{~mL}$ ). They reported a chronic retention rate of $16 \%$ and provide some surgical modifications to prevent this complication: reinforcing the back support of the neobladder with an omental flap, suspending the vaginal wall by the round ligaments or peritoneum, or suspending the pouch ventrally to the back of the rectus muscle ${ }^{[37]}$. Genital sparing surgery, when possible, is another alternative. Other possible causes are large capacity pouches due to excessive bowel segment length or even autonomic denervation of the urethra ${ }^{[36]}$.

After diagnosis of chronic retention, temporary measures involve manual reduction of the prolapse during voiding or the use of a pessary; surgical revision with ventral suspension of the pouch can also be tried ${ }^{[37]}$. Despite that, they might need intermittent catheterization. In a study by Ahmadi et al. ${ }^{[35]}, 9.5 \%$ of male patients needed at least one catheterization per day and 1 patient could not urinate without catheterization. Intermittent self-catheterization was most commonly started during the first post-operative year ${ }^{[35]}$.

Urinary retention can less frequently be due to subneovesical obstruction either by tumour recurrence, stenosis of the urethral anastomosis, or the urethra itself; the reported rates of these complications on one study were $1.1 \%, 1.2 \%$, and $0.9 \%$, respectively, and in this study all strictures were treated by endoscopy ${ }^{[16]}$. Therefore, patients with emptying failure should undergo urethrocystoscopy to identify these possible causes.

\section{Incontinence}

Urinary incontinence is a very subjective complication. Its rates in the literature vary between daytime (7\%$13 \%)$ and nighttime (14\%-43\%) and depend greatly on the type of questionnaire used. A more objective way of evaluating urinary incontinence is pad weight measurement, but it also lacks standardizing, and an alternative measure is the number of pads per day ${ }^{[35]}$. This heterogeneity plus the variety of possible procedures (such as cystoprostatectomy, pelvic exenteration, vaginal-sparing techniques, and nervesparing) hinders the comparison of different studies ${ }^{[38]}$. 
In a study by Ahmadi et al ${ }^{[35]}$, self-reported rates of daily urinary leakage reached $39.7 \%$, causing almost half the patients to need to wear at least 1 pad per day. Nocturnal leakage was even higher (54.7\%) with many patients needing diapers at night. Notably, most did not report bothersome skin irritation or body odor ${ }^{[35]}$. Daytime incontinence of $3 \%-43 \%$ and nighttime incontinence of $0 \%-42 \%$ were the rates reported in a systematic review of female cystectomized patients. Nerve sparing techniques seem to improve continence, but more studies are needed in this area ${ }^{[38]}$.

Continence rates improve over time for most patients for up to 2 years after surgery, as the neobladder capacity increases. If incontinence persists, treatment options are diverse, including anti-muscarinic agents like tolterodine, periurethral collagen injections, bulking agents, urethral slings or external artificial urinary sphincters, and even trans-obturator taping ${ }^{[39,40]}$.

\section{CONCLUSION}

Radical cystectomy with urinary diversion is undoubtedly the most difficult surgical procedure in urology. Complication rates are high in the early and late settings, with various complications described in the literature. There is an urgent need of standardizing complication reporting so different series can be comparable. This review highlighted some of the most common complications and their possible management options. Careful patient selection, thorough long-term follow-up and standardization of complication reporting are mandatory conditions to achieve successful outcomes.

\section{DECLARATIONS}

\section{Authors' contributions}

Made substantial contributions to conception and organization of the review and performed literature review: Laranjo Tinoco C, Lima E

\section{Availability of data and materials}

Not applicable.

\section{Financial support and sponsorship}

None.

\section{Conflicts of interest}

Both authors declared that there are no conflicts of interest.

\section{Ethical approval and consent to participate}

Not applicable.

\section{Consent for publication}

Not applicable.

\section{Copyright}

(C) The Author(s) 2021.

\section{REFERENCES}

1. Lee RK, Abol-Enein H, Artibani W, et al. Urinary diversion after radical cystectomy for bladder cancer: options, patient selection, and outcomes. BJU Int 2014;113:11-23. DOI PubMed

2. Nazmy M, Yuh B, Kawachi M, et al. Early and late complications of robot-assisted radical cystectomy: a standardized analysis by urinary diversion type. J Urol 2014;191:681-7. DOI PubMed

3. Shabsigh A, Korets R, Vora KC, et al. Defining early morbidity of radical cystectomy for patients with bladder cancer using a standardized reporting methodology. Eur Urol 2009;55:164-74. DOI PubMed 
4. Hautmann RE, de Petriconi RC, Volkmer BG. Lessons learned from 1,000 neobladders: the 90-day complication rate. J Urol 2010;184:990-4; quiz 1235. DOI PubMed

5. Hirobe M, Tanaka T, Shindo T, et al. Complications within 90 days after radical cystectomy for bladder cancer: results of a multicenter prospective study in Japan. Int J Clin Oncol 2018;23:734-41. DOI PubMed

6. Parekh DJ, Reis IM, Castle EP, et al. Robot-assisted radical cystectomy versus open radical cystectomy in patients with bladder cancer (RAZOR): an open-label, randomised, phase 3, non-inferiority trial. Lancet 2018;391:2525-36. DOI PubMed

7. Tanneru K, Jazayeri SB, Kumar J, et al. Intracorporeal versus extracorporeal urinary diversion following robot-assisted radical cystectomy: a meta-analysis, cumulative analysis, and systematic review. J Robot Surg 2020. DOI PubMed

8. Cai Z, Li H, Hu J, et al. Intracorporeal versus extracorporeal urinary diversion after robot-assisted radical cystectomy: a pooled analysis. Gland Surg 2021;10:706-20. DOI PubMed PMC

9. Cerantola Y, Valerio M, Persson B, et al. Guidelines for perioperative care after radical cystectomy for bladder cancer: Enhanced Recovery After Surgery (ERAS(®)) society recommendations. Clin Nutr 2013;32:879-87. DOI PubMed

10. Ramirez JA, McIntosh AG, Strehlow R, Lawrence VA, Parekh DJ, Svatek RS. Definition, incidence, risk factors, and prevention of paralytic ileus following radical cystectomy: a systematic review. Eur Urol 2013;64:588-97. DOI PubMed

11. Clifford TG, Katebian B, Van Horn CM, et al. Urinary tract infections following radical cystectomy and urinary diversion: a review of 1133 patients. World J Urol 2018;36:775-81. DOI PubMed

12. Ghoreifi A, Van Horn $\mathrm{CM}, \mathrm{Xu} \mathrm{W}$, et al. Urinary tract infections following radical cystectomy with enhanced recovery protocol: A prospective study. Urol Oncol 2020;38:75.e9-75.e14. DOI PubMed

13. Parker WP, Toussi A, Tollefson MK, et al. Risk factors and microbial distribution of urinary tract infections following radical cystectomy. Urology 2016;94:96-101. DOI PubMed

14. Shariat SF, Karakiewicz PI, Palapattu GS, et al. Outcomes of radical cystectomy for transitional cell carcinoma of the bladder: a contemporary series from the Bladder Cancer Research Consortium. J Urol 2006;176:2414-22; discussion 2422. DOI PubMed

15. Shimko MS, Tollefson MK, Umbreit EC, Farmer SA, Blute ML, Frank I. Long-term complications of conduit urinary diversion. $J$ Urol 2011;185:562-7. DOI PubMed

16. Hautmann RE, de Petriconi RC, Volkmer BG. 25 years of experience with 1,000 neobladders: long-term complications. $J$ Urol 2011;185:2207-12. DOI PubMed

17. Krajewski W, Piszczek R, Krajewska M, Dembowski J, Zdrojowy R. Urinary diversion metabolic complications - underestimated problem. Adv Clin Exp Med 2014;23:633-8. DOI PubMed

18. Marien T, Robles J, Kammann TM, et al. Characterization of urolithiasis in patients following lower urinary tract reconstruction with intestinal segments. J Endourol 2017;31:217-22. DOI PubMed

19. Muto G, Collura D, Simone G, et al. Stapled orthotopic ileal neobladder after radical cystectomy for bladder cancer: Functional results and complications over a 20-year period. Eur J Surg Oncol 2016;42:412-8. DOI PubMed

20. Browne E, Lawrentschuk N, Jack GS, Davis NF. A systematic review and meta-analysis of the long-term outcomes of ileal conduit and orthotopic neobladder urinary diversion. Can Urol Assoc J 2021;15:E48-57. DOI PubMed PMC

21. Lobo N, Dupré S, Sahai A, Thurairaja R, Khan MS. Getting out of a tight spot: an overview of ureteroenteric anastomotic strictures. Nat Rev Urol 2016;13:447-55. DOI PubMed

22. Ahmadi N, Ashrafi AN, Hartman N, et al. Use of indocyanine green to minimise uretero-enteric strictures after robotic radical cystectomy. BJU Int 2019;124:302-7. DOI PubMed

23. Schöndorf D, Meierhans-Ruf S, Kiss B, et al. Ureteroileal strictures after urinary diversion with an ileal segment-is there a place for endourological treatment at all? J Urol 2013;190:585-90. DOI PubMed

24. Scherzer ND, Greenberg JW, Shaw EJ, Silberstein JL, Thomas R, Krane LS. Robotic vs. open surgical management of ureteroenteric anastomotic strictures: technical modifications to enhance success. J Robot Surg 2020;14:615-9. DOI PubMed

25. Rosales A, Emiliani E, Salvador JT, et al. Laparoscopic management of ureteroileal anastomosis strictures: initial experience. Eur Urol 2016;70:493-8. DOI PubMed

26. Narang SK, Alam NN, Campain NJ, et al. Parastomal hernia following cystectomy and ileal conduit urinary diversion: a systematic review. Hernia 2017;21:163-75. DOI PubMed

27. Rodriguez Faba O, Rosales A, Breda A, et al. Simplified technique for parastomal hernia repair after radical cystectomy and ileal conduit creation. Urology 2011;77:1491-4. DOI PubMed

28. Liedberg F, Kollberg P, Allerbo M, et al. Preventing parastomal hernia after ileal conduit by the use of a prophylactic mesh: a randomised study. European Urology 2020;78:757-63. DOI PubMed

29. Til H, Segarra Tomás J, De la Torre Holguera P, Monllau Font V, Palou Redorta J, Villavicencio Mavrich H. Rotura recurrente de neovejiga ileal: manejo conservador. Actas Urológicas Españolas 2007;31:279-84. DOI PubMed

30. Msezane L, Reynolds WS, Mhapsekar R, Gerber G, Steinberg G. Open surgical repair of ureteral strictures and fistulas following radical cystectomy and urinary diversion. J Urol 2008;179:1428-31. DOI PubMed

31. Lee DH, Song W. Surgical outcomes of transvaginal neobladder-vaginal fistula repair after radical cystectomy with ileal orthotopic neobladder: a case-control study. Cancer Manag Res 2020;12:10279-86. DOI PubMed PMC

32. Rapp DE, O'connor RC, Katz EE, Steinberg GD. Neobladder-vaginal fistula after cystectomy and orthotopic neobladder construction. BJU Int 2004;94:1092-5; discussion 1095. DOI PubMed

33. Carlos D, Abraham N, Zhou TC, Hung M. Transvaginal repair of neobladder vaginal fistula with Martius flap. Int Braz J Urol 2020;46:864-6. DOI PubMed PMC

34. Wilson A, Pillay S, Greenwell T. How and why to take a Martius labial interposition flap in female urology. Transl Androl Urol 
2017;6:S81-7. DOI PubMed PMC

35. Ahmadi H, Skinner EC, Simma-Chiang V, et al. Urinary functional outcome following radical cystoprostatectomy and ileal neobladder reconstruction in male patients. J Urol 2013;189:1782-8. DOI PubMed

36. Zahran MH, Eldemerdash Y, Taha DE, Sheir K, Shaaban AA, Ali-El-Dein B. Chronic urinary retention after radical cystectomy and orthotopic neobladder in women: Risk factors and relation to time. Urol Oncol 2017;35:671.e11-6. DOI PubMed

37. Ali-el-dein B, Gomha M, Ghoneim MA. Critical evaluation of the problem of chronic urinary retention after orthotopic bladder substitution in women. J Urol 2002;168:587-92. PubMed

38. Smith AB, Crowell K, Woods ME, et al. Functional outcomes following radical cystectomy in women with bladder cancer: a systematic review. Eur Urol Focus 2017;3:136-43. DOI PubMed

39. Jindal T, Mukherjee S, Mandal SN, Karmakar D. Transobturator taping for the treatment of incontinence after neobladder reconstruction. Female Pelvic Med Reconstr Surg 2013;19:245-6. DOI PubMed

40. Zahran MH, Harraz AM, Taha DE, Nabeeh H, El Hefnawy AS, Ali-El-Dein B. The short-term effects of tolterodine on nocturnal incontinence after ileal orthotopic neobladder: a randomised crossover placebo-controlled study. BJU Int 2019. DOI PubMed 